\title{
Synthesis of hexadecylbenzene sulfonate and its application in alkali-free flooding systems
}

\author{
Changxin Shi $\cdot$ Guangzhi Liao $\cdot$ Weidong Liu
}

Received: 28 August 2012/Accepted: 8 April 2013/Published online: 17 April 2013

(c) The Author(s) 2013. This article is published with open access at Springerlink.com

\begin{abstract}
Hexadecylbenzene sulfonate was synthesized using aluminum trichloride catalyzed Friedel-Crafts reaction with hexadecyl linear internal olefin to benzene, sulfonated by chlorosulfonic acid, and neutralized with sodium hydroxide. The structure of the synthesized hexadecylbenzene sulfonate was characterized by infrared spectroscopy and mass spectroscopy. Surfactant mixtures were formulated by the synthesized hexadecylbenzene sulfonate and Kelamayi petroleum sulfonate. The results showed that the surfactant mixtures could reduce the interfacial tension to ultra-low values in the presence as well as the absence of polymer, at a very low concentration. The surfactant/polymer systems had a good thermal stability. The recovery factor was $59.85 \%$ of original oil in place when the surfactant/polymer slug size was 0.3 pore volume of the core, compared with $45.59 \%$ in case of water flood.
\end{abstract}

Keywords Synthesis - Hexadecylbenzene - Sulfonate · Alkali-free $\cdot$ Interfacial $\cdot$ Tension

\section{Introduction}

Much oil remained trapped in the pores and capillaries of petroleum reservoir rock after secondary recovery. In order

C. Shi $(\square) \cdot$ G. Liao · W. Liu

Institute of Porous Flow and Fluid Mechanics,

Chinese Academy of Science, Langfang 065007,

Hebei, China

e-mail: cxshi@qq.com

G. Liao

Exploration and Development Company,

CNPC, Dongcheng, Beijing 100007, China to drive them out, the capillary number should be maximized by either increasing viscosity or reducing interfacial tension (Al-Sahhaf et al. 2002); it was found that the residual oil saturation might be close to zero, if the interfacial tension between oil and water was reduced to ultra low $\left(<10^{-2} \mathrm{~m} / \mathrm{Nm}\right)$ (Foster 1973). In the past, alkali-surfactant-polymer (ASP) flooding method was wildly used in China (Cui et al. 2011). Alkali and surfactant had a synergistic effect in reducing the interfacial tension (Liu et al. 2007). However, the addition of alkali will cause the scaling problems, which will affect formation permeability, decrease pump inspection period ( $\mathrm{Li}$ et al. 2012), and lead to polymer viscosity reduction. To avoid those side effects, organic alkali has been proposed; nevertheless, it is not cheap (Guerra et al. 2007). Petroleum sulfonate is a widely available, inexpensive oil displacement agent. Many researchers have reported its usage in the oil recovery (Chen et al. 2012; Hou et al. 2001). It was found that (Yang et al. 2005) hexadecylbenzene sulfonate could reduce the interfacial tension ultra-low values at low alkali concentrations when a phenyl group is located near the center of the alkyl chain.

In this study, we prepared hexadecylbenzene sulfonate by using hexadecyl linear internal olefin and benzene, sulfonating, neutralization, then combined with petroleum sulfonate used as oil displacement agents without using alkali.

\section{Experimental}

Apparatus and reagents

Hexadecyl internal olefin was obtained from Shanghai Qicheng Industrial Co., Ltd. Kelamayi petroleum sulfonate 
(KPS) was provided by the Kelamayi chemical plant, whose effective content is $44.56 \%$. Hydrolyzed polyacrylamide (HPAM) was supplied by Dagang Bohong Petroleum Chemical Company. The polymer has a degree of hydrolysis of $28 \%$, with the relative molecular weight of $2.5 \times 10^{7}$. The water used in this experiment is the formation water. The total dissolved solid is $13,454 \mathrm{mg} / \mathrm{L}$, containing $350 \mathrm{mg} / \mathrm{L}$ of calcium ion and $180 \mathrm{mg} / \mathrm{L}$ of magnesium ion. The reservoir temperature is $53.2^{\circ} \mathrm{C}$. The crude oil used for the present study was offered from the third block of Dagang oilfield Gangxi area in China. Aluminum trichloride, benzene, and sodium hydroxide were purchased from Tianjin Hongyan chemical reagent Company (AR grade) and Chlorosulfonic acid (AR grade) was purchased from West Asia chemical reagent Company. Measurement of FT-IR spectra was recorded on a Nicolet 8700 FT-IR spectrometer using $\mathrm{KBr}$ pellets.

MS analysis was performed using a Waters xevog2qtof mass spectrometer.

Measurements of the interfacial tension were made by the spinning-drop technique at $53.2^{\circ} \mathrm{C}$ using a data physics interfacial tensiometer.

\section{Synthesis}

The synthetic route of hexadecylbenzene sulfonate was as follows: (Scheme 1).

\section{Synthesis of hexadecylbenzene sulfonate}

Aluminum trichloride $(0.2 \mathrm{~mol})$ was quickly added into a round bottom flask with $50 \mathrm{ml}$ of benzene. Then, $0.05 \mathrm{~mol}$ of the hexadecyl internal olefin was added drop by drop using a mechanical stirrer at a speed of about $500 \mathrm{rpm}$. After the addition of the hexadecyl internal olefin, the system was kept at $65^{\circ} \mathrm{C}$ for $5 \mathrm{~h}$ to ensure a complete reaction. After the reaction was finished, dilute hydrochloric acid was added to hydrolyze the aluminum trichloride. The mixture then was transferred to a funnel of $250 \mathrm{~cm}^{3}$ and allowed to separate. The upper layer was collected and after vacuum distillation, the hexadecylbenzene was obtained. The synthesized hexadecylbenzene was put into a flask with a mechanical stirring $(200 \mathrm{rpm})$ in the ice-water bath. The solution of chlorosulfonic acid in dichloromethane was dropped slowly from a funnel into the flask with the hexadecylbenzene/chlorosulfonic acid molar ratio kept at 1/1.1. After that the system was kept at room temperature for $2 \mathrm{~h}$ to continue the reaction. Then, the $\mathrm{pH}$ of the reaction mixture was maintained $7-8$ by adding $30 \%$ sodium hydroxide solution. Petroleum ether (bp 60-90 ${ }^{\circ} \mathrm{C}$ ) was used to extract most of the unreacted hexadecylbenzene. The synthesized hexadecylbenzene sulfonate was dried and was next washed with ethanol. The final product was obtained after evaporation of the ethanol.

\section{Results and discussion}

Identification of structures

\section{Infrared spectroscopy}

The infrared spectrum of the product is shown in Fig. 1 where the adsorption at 1,199, 1,044, 1,013, and $609 \mathrm{~cm}^{-1}$ are the characteristic peaks of the sulfonic group; the adsorption at $721 \mathrm{~cm}^{-1}$ refers to stretching vibration of the $\mathrm{C}-\left(\mathrm{CH}_{2}\right) \quad n-\mathrm{C} \quad(n>4)$; the adsorption at 2,924 and $2,854 \mathrm{~cm}^{-1}$ refer to stretching vibration of the methylene; the adsorption at 1,462 and $1,602 \mathrm{~cm}^{-1}$ are due to the stretching vibration of benzene skeleton, peaks at 837 and $690 \mathrm{~cm}^{-1}$ are assigned to a p-substituted benzene ring vibration.

\section{ESI-MS spectrum}

The product was analyzed by ESI-MS in negative mode. The spectrum is shown in Fig. 2.

The ion at $\mathrm{m} / \mathrm{z} 381.24$ corresponds to $[\mathrm{M}-\mathrm{Na}]^{-}$of the hexadecylbenzene sulfonate, peaks at $\mathrm{m} / \mathrm{z} 382.24,383.24$, and 384.24 are due to the isotopic peaks of the molecular
Scheme 1 Preparation of the hexadecylbenzene sulfonate

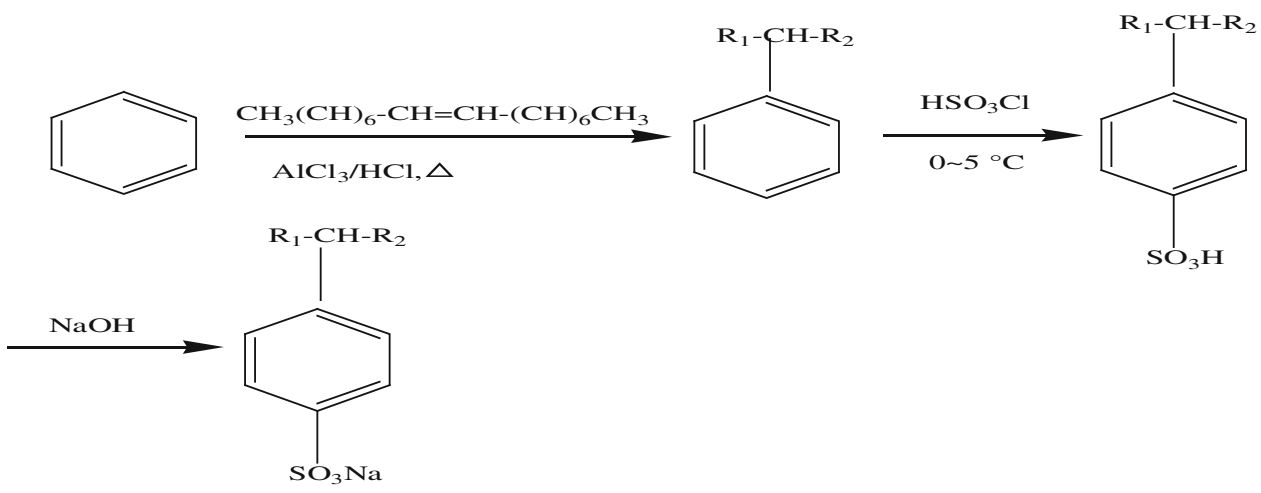




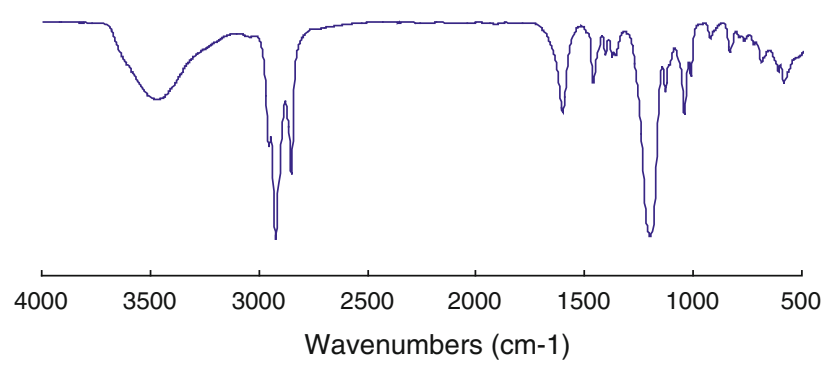

Fig. 1 Infrared spectrogram of hexadecylbenzene

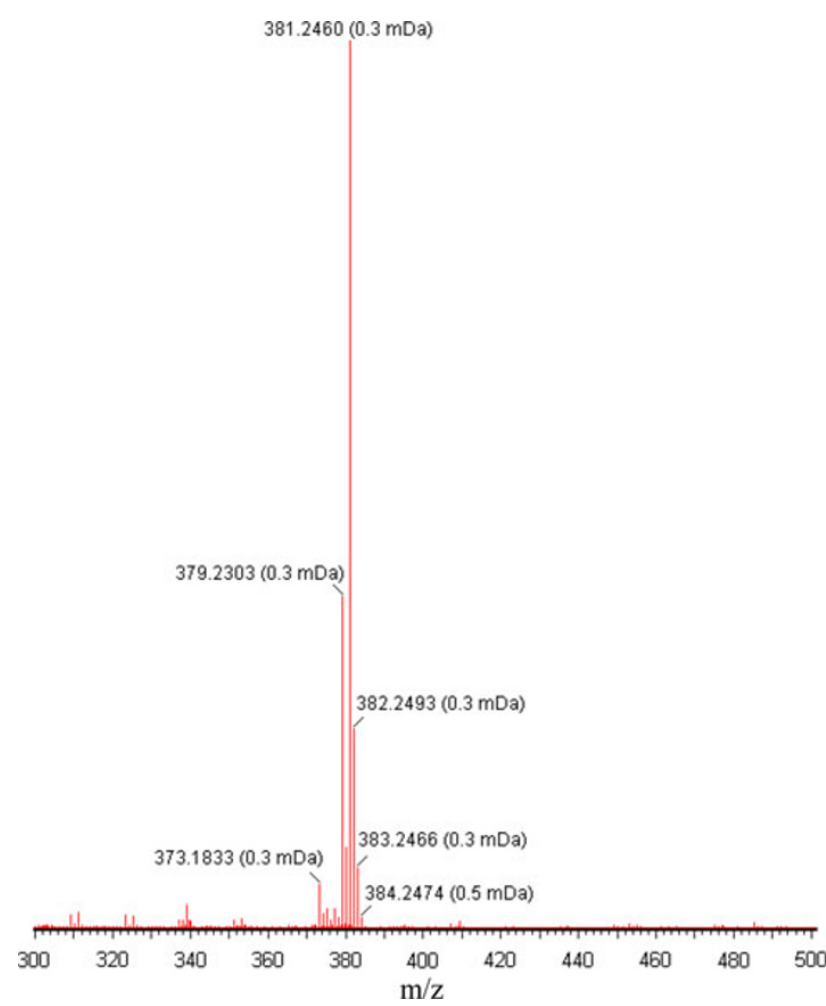

Fig. 2 MS spectrum of hexadecylbenzene sulfonate

ions. The ion at $\mathrm{m} / \mathrm{z} 379.23$ may be the deprotonated molecular ion.

\section{Measurement of oil-water interfacial tensions}

It could be obtained from Fig. 3 that single KPS solutions or hexadecylbenzene sulfonate could not reduce the interfacial tension to ultra-low values. So, the mixed surfactant systems were investigated. The two surfactants concentrations in the solutions were changed from 0.025 to $0.3 \%$ (mass ratio), respectively, the lowest interfacial tension values between oil and mixed surfactant solutions are shown in Table 1.

Table 1 showed the binary mixtures of surfactants had synergistic effects. The minimum interfacial tension value

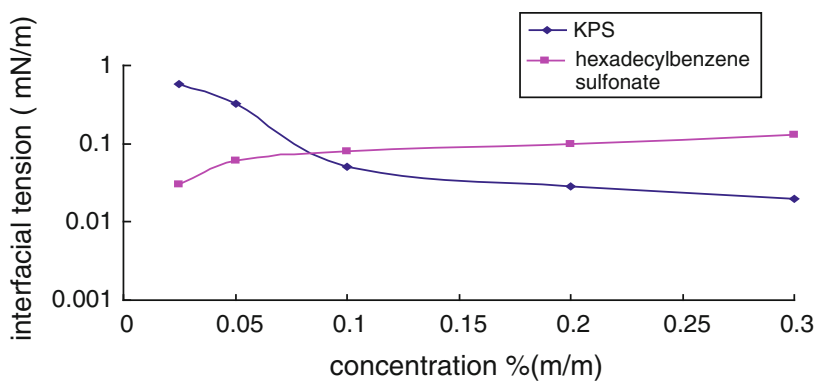

Fig. 3 The lowest dynamic interfacial tension values at different surfactant concentration

Table 1 The lowest interfacial tension value of the surfactant mixtures

\begin{tabular}{lll}
\hline KPS concentration $(\%)$ & $\begin{array}{l}\text { Hexadecylbenzene } \\
\text { sulfonate }(\%)\end{array}$ & $\begin{array}{l}\text { Interfacial } \\
\text { tension } \mathrm{mN} / \mathrm{m}\end{array}$ \\
\hline 0.1 & 0.1 & $7.92 \times 10^{-2}$ \\
0.1 & 0.05 & $1.50 \times 10^{-1}$ \\
0.05 & 0.1 & $6.42 \times 10^{-2}$ \\
0.05 & 0.05 & $2.42 \times 10^{-2}$ \\
0.025 & 0.025 & $1.31 \times 10^{-3}$ \\
0.02 & 0.01 & $4.38 \times 10^{-2}$ \\
0.015 & 0.015 & $2.45 \times 10^{-4}$ \\
0.01 & 0.02 & $1.31 \times 10^{-3}$ \\
0.01 & 0.01 & $1.50 \times 10^{-2}$ \\
0.005 & 0.01 & $1.05 \times 10^{-3}$ \\
\hline
\end{tabular}

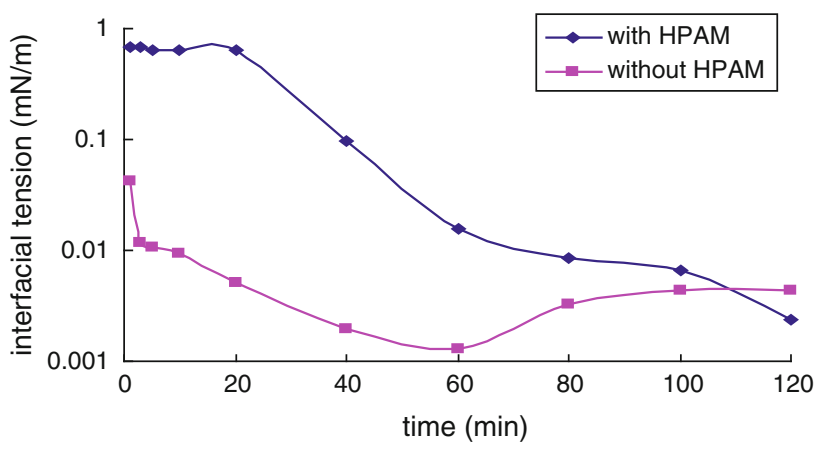

Fig. 4 Dynamic interfacial tension values at different times

was $2.45 \times 10^{-4} \mathrm{mN} / \mathrm{m}$, when the surfactant concentration was $0.015 \%$ KPS $+0.015 \%$ hexadecylbenzene sulfonate; the mixed surfactant system could lower the oil/water interfacial tension to ultra low even when the total concentration of surfactant is $0.015 \% \quad(0.005 \%$ KPS + $0.01 \%$ hexadecylbenzene sulfonate).However, in view of the effect of adsorption, the surfactant mixture that contained $0.025 \%$ KPS $+0.025 \%$ hexadecylbenzene sulfonate was used as oil displacement agent. To investigate the presence of HPAM on the interfacial tension between the 
Fig. 5 Dynamic interfacial tension values at different aging time

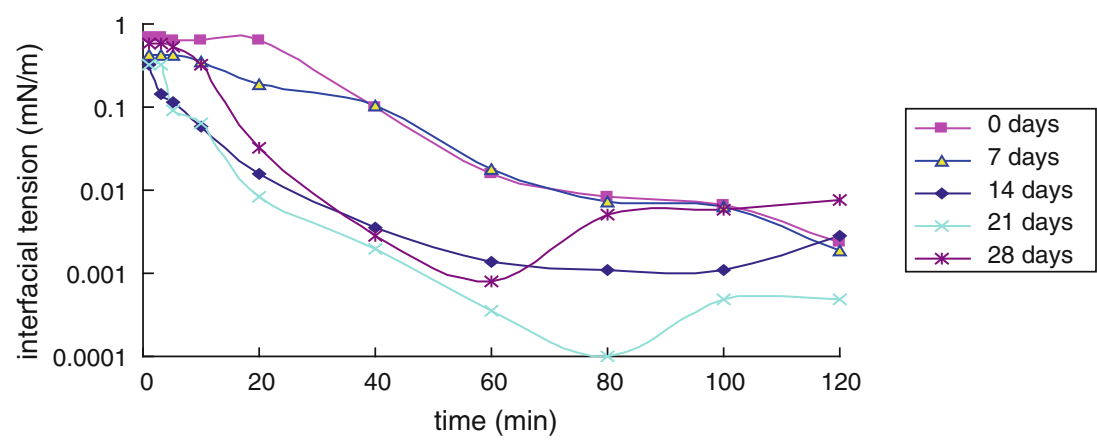

Fig. 6 Relation between injection fluid porous volume and oil recovery, water cut

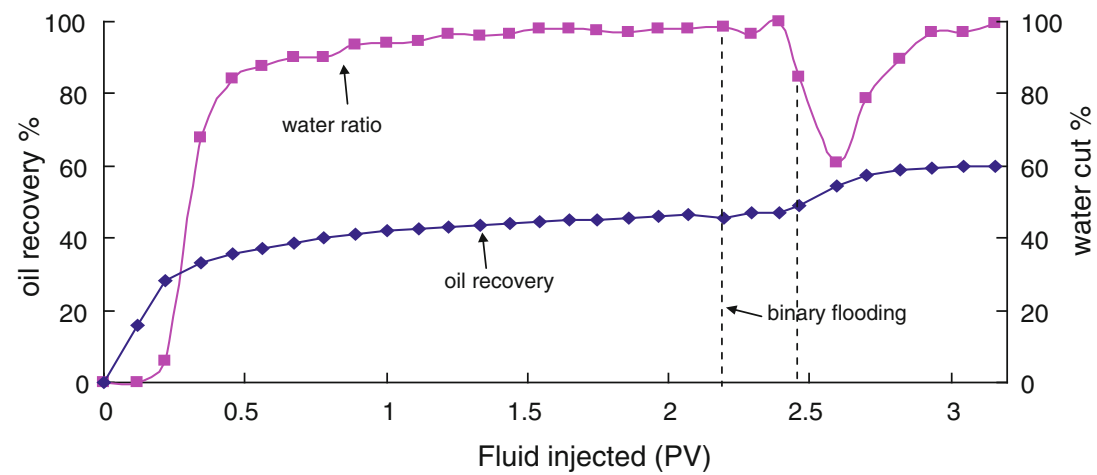

It is seen from Fig. 5 that the surfactant-polymer systems could reduce the interfacial tension to ultra-low values even when placed 28 days at $53.2^{\circ} \mathrm{C}$. The interfacial tension was first gradually decreased with time to the lowest value (near $10^{-5} \mathrm{mN} / \mathrm{m}$ ) at 21 days and then increased. Also, the time to reach ultra low was shortened from 78 to $15 \mathrm{~min}$, and then was prolonged to $25 \mathrm{~min}$. This is because the dissolved oxygen in the surfactant-polymer solution accelerated thermal degradation of the polymer, which results in loss of viscosity, thus, which speeds up the transfer rate at the interface and shortens the time that needs to reach the ultra-low values. The existence of oxygen also causes the degradation of the surfactant, making the interfacial tension rise and prolongation of time (Ma et al. 2008).

\section{Sand-pack core flooding test}

A sand-pack core flooding test was performed at $53.2{ }^{\circ} \mathrm{C}$ in sand-packed tube $(30 \mathrm{~cm}$ long and $1.80 \mathrm{~cm}$ in diameter). Its permeability to water was $876 \mathrm{mD}$ and the porous volume was $26.5 \mathrm{ml}$. The core was first saturated with the desired crude oil sample, and then flooded with water until water cut was more than $98 \%$. Next, $0.3 \mathrm{PV}$ of polymer/surfactant $(1,500 \mathrm{mg} / \mathrm{L}$ HPAM $+0.025 \% \mathrm{KPS}+0.025 \%$ hexadecylbenzene sulfonate) was injected at $0.6 \mathrm{~mL} / \mathrm{min}$. After polymer/surfactant flooding, water was injected until water cut was more than $98 \%$. The results are given in Fig. 6.

As shown in Fig. 6, the water ratio of the effluent increased rapidly with the increase of the oil recovery after solutions. The results are shown in Fig. 5. 
the injection of the polymer-surfactant mixture; the water ratio first decreased and then again increased and some of the residual oil was also recovered during this process. The final oil recovery was $59.85 \%$, and the enhanced oil recovery by the surfactant-polymer flooding increased the ultimate recovery by $14.26 \%$.

\section{Conclusions}

Hexadecylbenzene sulfonate was synthesized and characterized. When mixed with Kelamayi petroleum sulfonate, the surfactant mixtures could reduce Dagang crude oil/ connate water interfacial tension to a magnitude of $10^{-3}$ $10^{-4} \mathrm{mN} / \mathrm{m}$ at a very low concentration in the presence or absence of polymer. The surfactant-polymer system had a good thermal stability. Oil displacement tests using sandpacked core showed that $14.2 \%$ of the additional oil could be obtained over the water flood by the SP flooding.

Open Access This article is distributed under the terms of the Creative Commons Attribution License which permits any use, distribution, and reproduction in any medium, provided the original author(s) and the source are credited.

\section{References}

Al-Sahhaf T, Suttar Ahmed A, Elkamel A (2002) Producing ultra low interfacial tension at the oil/water interface. Petrol Sci Technol 20(7\&8):773-778
Chen T, Zhang G, Ge J (2012) Dynamic interfacial tension between Gudao heavy oil and petroleum sulfonate/HPAM complex systems. Petrol Sci Technol 30(4):1417-1423

Cui Z, Song H, Yu J, Jiang J, Wang F (2011) Synthesis of $N$-(3Oxapropanoxyl)dodecanamide and its application in surfactantpolymer flooding. J Surfact Deterg 14:317-324

Foster W (1973) A low-tension water flooding process. J Pet Technol 25(2):205-210

Guerra E, Valero E, Rodríguez D, Gutierrez L, Castillo M, Espinoza J, Granja G (2007) Improved ASP Design Using Organic Compound-Surfactant-Polymer (OCSP) for La Salina Field, Maracaibo Lake. In: SPE 107776 presented at Latin American \& Caribbean Petroleum Engineering Conference, Buenos Aires, Argentina, 15-18 April 2007

Hou Z, Li Z, Wang H (2001) Ultra-low interfacial tension in oilwater-mixed surfactant systems. J Disper Sci Technol 22(2-3):255-299

Li N, Zhang G, Ge J, Zhang L, Liu X (2012) Ultra-low interfacial tension between heavy oil and betaine-type amphoteric surfactants. J Disper Sci Technol 33(2):258-264

Liu Q, Dong M, Ma S, Tu Y (2007) Surfactant enhanced alkaline flooding for Western Canadian heavy oil recovery. Colloid Surface A 293(1-3):63-71

Ma T, Shao H, Wang H, Jiang P, Wei T, Tang D (2008) A laboratory study of the composite two component oil displacement system without alkali. Chin Offshore Oil Gas 20(1):40-43

Yang J, Qiao W, Li Z, Cheng L (2005) Effects of branching in hexadecylbenzene sulfonate isomers on interfacial tension behavior in oil/alkali systems. Fuel 84:1607-1611 